Gut, 1988, 29, 1613-1617

Case report

\title{
Treatment of end stage chronic intestinal pseudo- obstruction by subtotal enterectomy and home parenteral nutrition
}

\author{
M M MUGHAL AND M H IRVING \\ From the Hope Hospital, University of Manchester School of Medicine, Salford
}

SUMMARY Three cases are described of end stage chronic intestinal pseudo-obstruction successfully treated by subtotal enterectomy and home parenteral nutrition (HPN). In all three patients prior use of drugs to stimulate gastrointestinal motility, antibiotics to suppress bacterial overgrowth and surgical bypass of dilated bowel had failed to alleviate the symptoms of chronic intestinal obstruction. Similarly parenteral nutrition combined with restriction of oral intake, although improving nutrition did not relieve symptoms. The treatment of end stage chronic pseudoobstruction should relieve intestinal obstruction and correct nutritional deficiency. In our experience this is best achieved by subtotal enterectomy, restoration of continuity by end-to-end anastomosis and total parenteral nutrition.

Chronic intestinal pseudo-obstruction is difficult to diagnose and treat. Pharmacological manipulation, enteral and parenteral nutrition, and surgery in the form of bypass, resection or the creation of venting stomas have all been used with varying degrees of success. Pharmacological manipulation is rarely successful and parenteral nutrition, whilst of value in restoring nutritional status, ${ }^{1}$ does not relieve vomiting, abdominal pain or distension ${ }^{2}$ because gastrointestinal secretions continue to pour into the aperistaltic bowel. Bypass operations are only beneficial if an affected segment can be excluded and even then bacterial overgrowth in the blind loop so created may mar the results.

There are only a handful of reports in the literature of radical bowel resection for the management of pseudo-obstruction and none to our knowledge of near total enterectomy. We report our experience with three patients treated by radical enterectomy followed by longterm home parenteral nutrition (HPN).

Address for correspondence: Professor M H Irving, University Department of Surgery, Hope Hospital, Eccles Old Road, Salford M6 8HD.

Received for publication 23 May 1988.
Case 1

A 21 year old merchant seaman was referred to Hope Hospital in 1976 with a 12 year history of episodic colicky abdominal pain and vomiting for which he had previously undergone numerous investigations and many exploratory operations. Barium studies undertaken on admission showed a dilated duodenum proximal to a 'stricture' in the second part of the duodenum (Fig. 1). At laparotomy no stricture was found but the duodenojejunal flexure appeared to be kinked by adhesions. It was noted that the proximal small bowel was dilated. A gastrojejunostomy was carried out. After operation his symptoms continued unabated and subsequently he developed steatorrhoea. Jejunal biopsy showed infiltration of the lamina propria with lymphocytes and plasma cells; there was no mucosal atrophy. Glucose and xylose tolerance tests were normal as were pancreatic isotope, ultrasonic and computed tomography (CT) scans. The secretin-pancreozymin test showed a decreased volume of pancreatic secretions with a normal bicarbonate concentration raising the possibility of ductal obstruction but this was excluded by a normal duct on ERCP. A $\mathrm{C}^{14}$ glycocholate test 


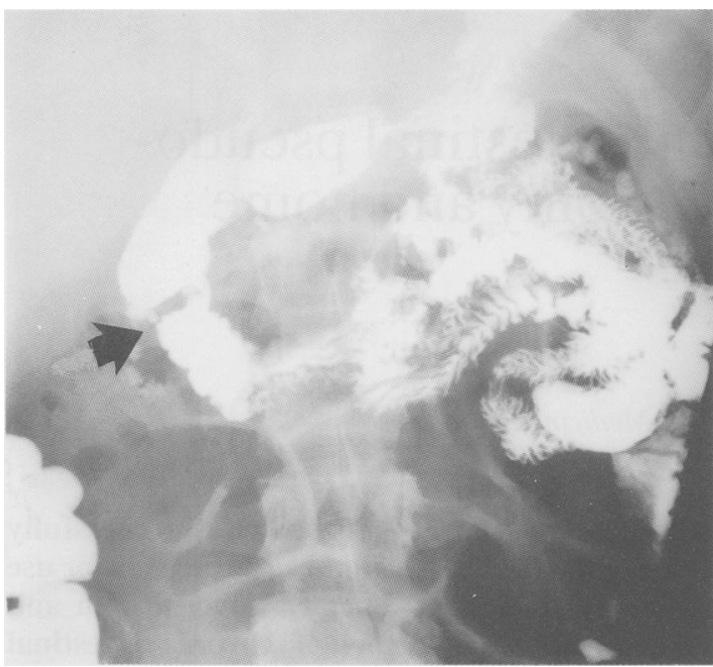

Fig. 1 Barium meal in case I showing a dilated duodenum proximal to an apparent stricture (arrowed) in the second part of the duodenum.

showed evidence of bacterial overgrowth. The daily faccal fat excretion was raised at $75 \mathrm{mmol} / \mathrm{day}$ (normal $10-20 \mathrm{mmol} /$ day). He was treated with a combination of low fat diet, cimetidine, antibiotics and pancreatic supplements without benefit. Repeat barium follow through 18 months later showed marked dilatation and stasis of the small bowel (Fig. 2) and a further laparotomy was done in May 1978. The entire small bowel was thickened and dilated and there were fleshy mesenteric lymph nodes. Adhesions were divided, and biopsies taken of the full thickness of the small bowel and a mesenteric lymph node. Histological examination of the former revealed only lymphoid hyperplasia with reactive hyperplasia in the lymph node. Silver staining for myenteric plexi was unfortunately not done.

Because of continuing weight loss he was started on parenteral nutrition and his oral intake was reduced to relieve abdominal distension. He was also sequentially treated with metoclopramide, bethanechol, atropine, propranolol, and indomethacin. Although his weight stabilised on parenteral nutrition there was little improvement in his gastrointestinal symptoms. He was weaned off parenteral nutrition after three weeks and started on an elemental diet and discharged. He was readmitted three months later with a further weight loss of $2.5 \mathrm{~kg}$ and serum albumin of $27 \mathrm{~g} / 1$. He was started on home parenteral nutrition (HPN) and restored to near normal weight after two months. Unfortunately, he was frequently readmitted with increasingly severe abdominal pain, gross distension, and vomiting

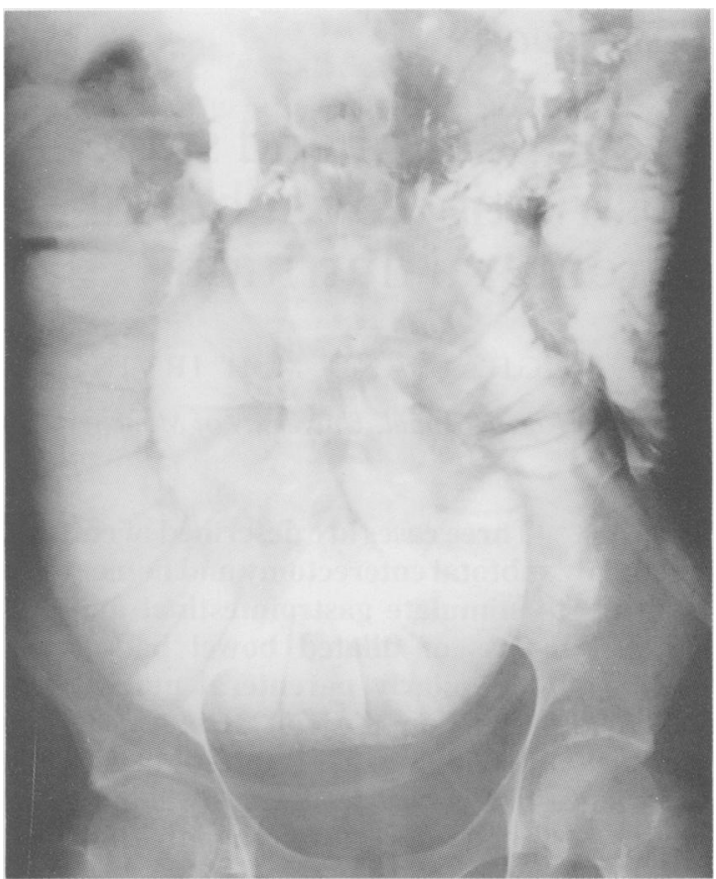

Fig. 2 Barium follow through in case 1 after deterioration of symptoms, showing marked dilatation and stasis of the proximal small bowel.

requiring permanent nasogastric intubation. A laparotomy was performed which showed marked distension of the small bowel. A Foley-catheter gastrostomy and a split ileostomy were performed to vent the proximal and distal bowel and a further full thickness biopsy was taken of the small bowel. This showed dense infiltration by lymphoid tissue and subtotal villous atrophy. Diffuse lymphocytic lymphoma was excluded by a normal bone marrow and trephine and normal serum, salivary and jejunal immunoglobulins. A diagnosis of chronic idiopathic pseudo-obstruction was therefore made by exclusion of other causes of obstruction.

The gastrostomy controlled his vomiting but the ileostomy drained very little and did not relieve his abdominal distension and pain. As there was no sign of restoration of intestinal motility it was decided to remove the amotile bowel. A further laparotomy was done and all the bowel from $20 \mathrm{~cm}$ distal to the duodenojejunal flexure to midtransverse colon was resected, continuity being restored by an end to end jejuno colic anastomosis. He made an uneventful recovery and was able to lead an active pain free life on home parenteral nutrition. He was particularly pleased at being able to eat and drink freely without inducing pain though he obviously had diarrhoea. 
Unfortunately he was killed in a road accident eight months later whilst driving his newly purchased sports car.

\section{Case 2}

A 55 year old West Indian man presented with features of subacute small bowel obstruction in October 1980, 16 months after being diagnosed as having scleroderma. The episode settled after a period of nasogastric suction and intravenous fluids but he had further similar episodes over the next three months. As the frequency of the episodes increased he began to lose weight, and he was treated with a combination of an elemental diet and metoclopramide. He was referred to Hope Hospital after failure of this regimen to control his symptoms.

On admission he was clinically well nourished, but had a grossly distended abdomen. Plain abdominal radiographs showed gaseous distension of the small and large bowel all the way round to the rectum. Manometry showed normal motility in the stomach but reduced motility in the duodenum and jejunum with only a slight improvement after the administration of metoclopramide. Skin and muscle biopsies showed the histological features of scleroderma and barium studies showed gross dilatation of the jejunum and proximal ileum with very slow transit. Lung function tests showed a deterioration of most indices. FEV 1.771, FVC 2.11, PEFR $250 \mathrm{l} / \mathrm{min}$, and $\mathrm{T}_{\mathrm{L}} \mathrm{CO} 17.11 \mathrm{ml} / \mathrm{min} / \mathrm{mmHg}$ (predicted values: 2.9 , $3.74,575$, and 24.9 respectively). Persistent treatment with enteral regimens failed to control his symptoms. He was therefore started on parenteral nutrition and $50 \mathrm{mg}$ of intramuscular prednisolone per day. Over the next two months his weight rose from $48.6 \mathrm{~kg}$ to $56.1 \mathrm{~kg}$ and his serum albumin from $29 \mathrm{~g} / \mathrm{l}$ to $41 \mathrm{~g} / \mathrm{l}$.

He continued to be troubled by nausea, vomiting, abdominal pain, and gross distension despite being restricted to only $500 \mathrm{ml}$ fluid orally. He was therefore trained in the techniques of HPN and discharged in June 1981 only to be readmitted a few weeks later. There was no improvement in his abdominal symptoms and he was confined to hospital producing large quantities of aspirate through his indwelling nasogastric tube. Laparotomy was carried out in October 1981 at which the entire small bowel was found to be dilated and aperistaltic. A subtotal enterectomy was done leaving $15 \mathrm{~cm}$ of jejunum anastomosed to $15 \mathrm{~cm}$ of terminal ileum. Histology of the excised bowel showed thickening of the bowel wall, stunting of the villi, and collagen deposition in the submucosa and the serosa consistent with a diagnosis of scleroderma. After this, his gastrointestinal symptoms improved considerably enabling him to go home. As with the first patient he was able to eat and drink without inducing pain and distension and led a relatively normal life despite the constraints of HPN. Unfortunately his respiratory condition deteriorated with progression of the scleroderma and he eventually died of cardiorespiratory failure in September, 1982.

\section{Case 3}

A 41 year old farm labourer presented to his local hospital in 1969 with a long history of intermittent epigastric pain and vomiting. Barium meal showed gross dilatation of the duodenum without an identifiable point of obstruction. A laparotomy confirmed this opinion and a duodenojejunostomy was done in the third part of the duodenum. He remained relatively well for seven years until 1977 when he was admitted as an emergency with acute onset of abdominal pain, distension and vomiting. Laparotomy showed the entire small bowel to be dilated without any mechanical cause and no procedure was performed. He continued to experience bouts of vomiting and a gastrojejunostomy was performed in July 1977. This failed to relieve the vomiting and at a further laparotomy in September 1977 the gastrojejunostomy was taken down and the previous duodenojejunostomy widened. Over the following year, the abdominal distension and pain became worse and led to a further laparotomy which merely confirmed the previous findings of dilated small bowel.

Between 1978 and 1984 a variety of antispasmodics and antibiotics were tried without success. He was then referred to the Nutrition Unit at Hope Hospital to be trained for HPN. Parenteral nutrition was started in May 1985 and his oral intake was limited. After two months there was little improvement. He continued with gross abdominal distension, vomiting, and constipation. The vomiting necessitated continued nasogastric intubation with an average daily aspirate of 4 to $5 \mathrm{I}$. In June 1985 a laparotomy was done which showed aperistaltic bowel and a subtotal enterectomy was performed with anastomosis of the first loop of the jejunum to the descending colon. Histological examination of the excised bowel showed normal myenteric plexuses with normal numbers of ganglion cells. Inner and outer layers of the muscularis propria of the normal bowel showed fibrosis. Although there was no vacuolisation of the smooth muscle cells, on balance the clinical and histological findings were consistent with hollow visceral myopathy.

After operation, the nasogastric aspirates remained high and a barium follow through showed duodenal dilatation with hold up at the anastomosis, which was nevertheless patent. This, along with the 


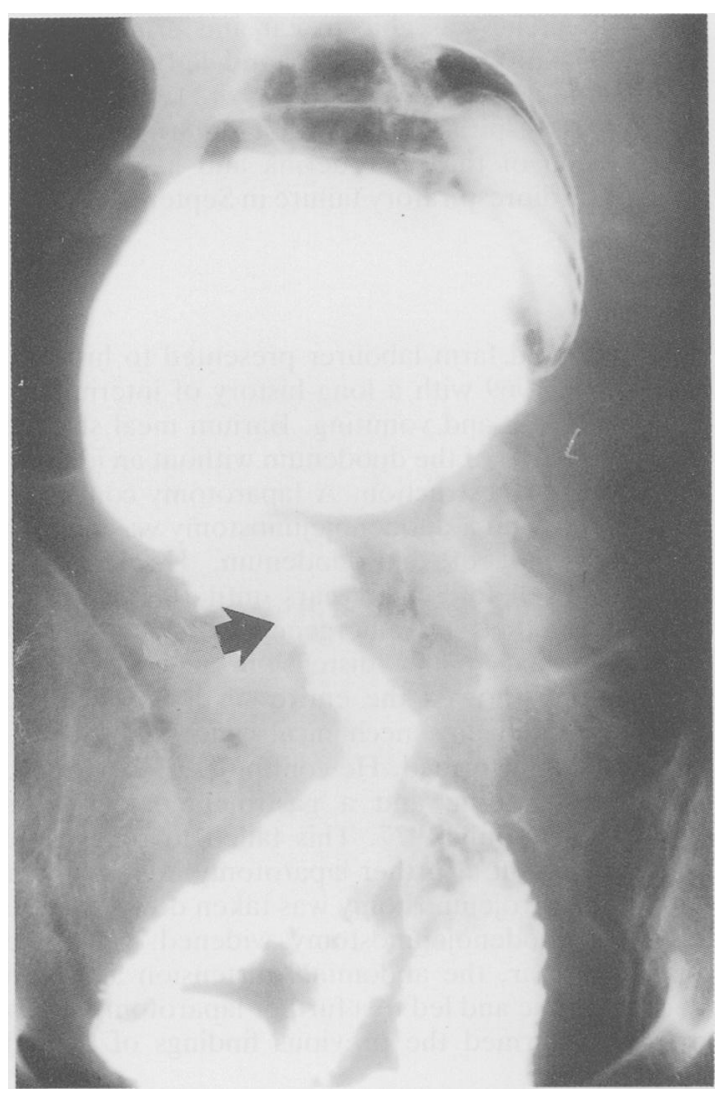

Fig. 3 Barium follow through after the penultimate operation in case 3 showing a widely patent duodeno-sigmoid anastomosis (arrowed) and a dilated duodenum proximally.

development of a low grade pyrexia prompted a further laparotomy on the clinical suspicion of a para-anastomotic abscess. No abscess was found, the anastomosis was taken down, more colon was resected and the duodenojejunal flexure anastomosed to the sigmoid colon. After this he continued to vomit intermittently although the anastomosis was again shown to be patent by endoscopy and barium studies (Fig. 3). Gastroscopy showed severe oesophagitis and oesophageal manometry showed duodenojejunal flexure anastomosed to the sigmoid colon. After this he continued to vomit intermittently although the anastomosis was again shown to be patent both by endoscopy and barium studies (Fig. 3). Gastroscopy showed severe oesophagitis and oesophageal manometry showed a hypotonic lower oesophageal sphincter and a complete absence of primary peristalsis in the distal twothirds of the oesophagus. He therefore underwent a further laparotomy at which the upper rectum was anastomosed to the anterior wall of the stomach. He was started on ranitidine and a Foley catheter gastrostomy was carried out to allow him to drain his stomach as and when necessary.

He resumed HPN and remained well for two years able to enjoy life at home and attend clubs and social events. He tolerated a light diet and required to use his gastrostomy tube occasionally. He remained in an excellent nutritional state throughout the two years, his only problem being a persistent pancytopaenia of uncertain origin for which he required regular blood transfusions. He was admitted in June 1987 with gastric haemorrhage after which he developed the adult respiratory distress syndrome and died.

\section{Discussion}

Enterectomy for pseudo-obstruction has been reported previously but the lengths of bowel resected are relatively small compared with those carried out in our cases. Paul and colleagues ${ }^{3}$ report a case of a 15 year old boy in whom all but $230 \mathrm{~cm}$ of small bowel was resected. Their case, as would be expected, did not require longterm parenteral nutrition. Schuffler and colleagues ${ }^{4}$ also report a case of radical resection in a case of visceral neuropathy leaving their patient with just less than $100 \mathrm{~cm}$ of small bowel. All three of our patients were left with less than $30 \mathrm{~cm}$ of small bowel, in addition to which, two also had a major colonic resection. In the two cases of chronic idiopathic pseudo-obstruction this radical procedure was done after a variety of different surgical procedures and medical treatment had failed. Only in the patient with scleroderma was massive resection performed at the first, and only, operation.

Intestinal pseudo-obstruction, particularly the chronic idiopathic variety, is a difficult condition to treat. Schuffler, ${ }^{5}$ in his exhaustive review of the subject points out the futility of attempts to stimulate gastrointestinal motility as the fundamental defect is a degeneration of either the muscle or the nerve plexuses. The only useful drugs are antibiotics which, by controlling bacterial overgrowth, may provide relief in some cases.

The failure to improve or restore gastrointestinal motility by pharmacological manipulation has led to the use of longterm parenteral nutrition in the management of this condition. Although Schuffler and colleagues ${ }^{2}$ found no improvement in symptoms in 10 patients treated with HPN over a four year period, Warner and Jeejeebhoy' reported an improvement in symptoms and in the nutritional status of 10 patients treated with a combination of parenteral nutrition and restriction of oral intake. Jeejeebhoy's group emphasise the importance of restricting oral intake in order to achieve relief of 
abdominal distension and vomiting. All three of our patients had a trial of parenteral nutrition with oral restriction without benefit. Indeed in case 3 the daily nasogastric aspirate remained 4-5 1 despite stopping all oral intake. It is difficult to envisage how even complete abstinence from oral intake can relieve vomiting when, as in our cases, the motility disorder affects predominantly the proximal gut. We conclude that a combination of parenteral nutrition and a reduced oral intake is only likely to be beneficial in patients with pseudo-obstruction affecting the distal bowel.

Some form of surgery is ultimately required in at least half of those with chronic idiopathic pseudoobstruction. ${ }^{6}$ For dilatation of the duodenum this is usually a bypass as resection of this part of the gastrointestinal tract is a major undertaking. Newton, ' however, questions the rationale for bypass in the absence of mechanical obstruction and Schuffler ${ }^{5}$ reports poor results from bypass illustrating this with the case of a patient who had undergone 34 operations, mainly bypasses without relief. This was certainly our experience in case 3 . The best operation for megaduodenum is duodenoplasty with resection of redundant duodenum. If this is not feasible it is better to opt for duodenojejunostomy which appears to provide better drainage than gastroenterostomy. ${ }^{6}$ Pitt and colleagues ${ }^{8}$ have used a combination of venting ostomy and HPN with some measure of success in 20 cases. In 18 of their patients the stoma was a gastrostomy. Our experience confirms the value of gastrostomy but more distal stomas in the ileum or colon merely act as sumps permitting overflow of the contents accumulating within distended, atonic, aperistaltic bowel.

We consider that in end stage pseudo-obstruction the only way to overcome the misery of gross abdominal distension, and the associated high gastric aspirates and chronic abdominal pain is by radical removal of this functionally dead bowel. This is achieved by resection of the majority of the affected bowel with end-to-end anastomosis. Until recently, this was not a practical proposition, but with the advent of safe longterm HPN these patients can simultaneously be freed from prolonged hospital stay and their unpleasant symptoms. The main complication of resection in our cases was a failure of the anastomosis to function despite demonstrable patency, which presumably is an indication of the fact that even the residual segments of bowel are aperistaltic. This was particularly manifest in our patient with hollow visceral myopathy who ultimately had to have a gastrorectal anastomosis. He also had a demonstrable loss of motility in his distal oesophagus and consequently developed severe oesophagitis which was only controlled by longterm high dose ranitidine and intermittent drainage of stomach contents through his gastrostomy.

We did not have any anastomotic leaks, and central venous catheter infection occurred in only one patient at the time we were setting up the home parenteral nutrition programme.

Our experience of subtotal enterectomy for intestinal pseudo-obstruction is small and follow up has only been prolonged in one case. It is unlikely that the operation improves the longterm prognosis of these patients who often have accompanying motility disorders in other systems. Excellent symptomatic relief, however, was obtained in all three cases by removal of the aperistaltic bowel. Although in the future other treatments such as somatostatin which effectively decreases gastrointestinal secretion may be of value, for the present subtotal enterectomy combined with HPN is a worthwhile treatment for patients with end stage intestinal pseudo-obstruction.

We are grateful to Dr W D W Rees for referring cases 1 and 2, and to Professor J Lennard-Jones and Dr I Dymock for referring case 3.

\section{References}

1 Warner E, Jeejeebhoy KN. Successful management of chronic intestinal pseudo-obstruction with home parenteral nutrition. JPEN 1979; 9: 173-8.

2 Schuffler MD, Rohrmann CA, Chaffee RL, et al. Chronic intestinal pseudo-obstruction: a report of 27 cases and a review of the literature. Medicine 1981; 60: 173-96.

3 Paul CA, Tomiyasu U, Mellinkoff SM. Nearly fatal pseudo-obstruction of the small intestine. A case report of its relief by sub-total resection of the small bowel. Gastroenterology 1961; 40: 698-704.

4 Schuffler MD, Leon SH, Krishnamurthy S. Intestinal pseudo-obstruction caused by a new form of visceral neuropathy; palliation by radical small bowel resection. Gastroenterology 1985; 89: 1152-6.

5 Schuffler MD, Deitch EA. Chronic idiopathic intestinal pseudo-obstruction. A surgical approach. Ann Surg 1980; 192: $752-61$

6 Anuras A, Shirazi S, Faulk DL, Gardner DG, Christensen J. Surgical treatment of familial visceral myopathy. Ann Surg 1979; 189: 306.

7 Newton WT. Radical enterectomy for hereditary megaduodenum. Arch Surg 1968; 96: 549-53.

8 Pitt HA, Mann LL, Berquist WE, et al. Chronic intestinal pseudo-obstruction. Management with total parenteral nutrition and a venting enterostomy. Arch Surg 1985; 120: $614-8$. 